\section{Did the Romans exploit uranium?}

Michael Vickers

Uranium Glass. By Ken Tomabechi. Iwanami Book Service Center, 2-3 Jinbocho Kanda Chiyoda-ku, Tokyo 101, Japan: 1995. Pp. 180. $¥ 7,000$.

KEN Tomabechi has spent his professional life in the Japanese atomic energy industry, but for many years his hobby has been the collection and study of objects made from a material known variously as Vase-

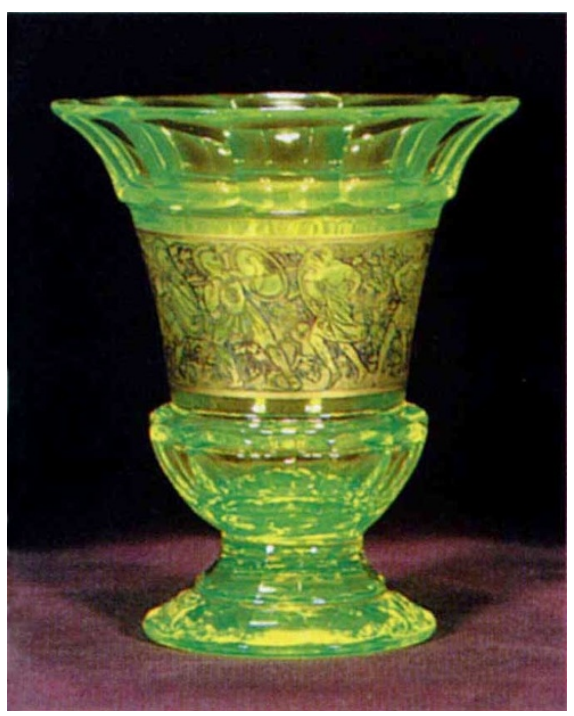

Uranium glass vase made by the famous Mose Glassworks in Czechoslovakia (c.1920).

line or uranium glass in English, Annagelb or Annagrün in German, and verre canari in French. Vases, candlesticks, ashtrays, clocks, drinking vessels and even jewellery were fashioned in this curious medium (which is fluorescent under ultraviolet light) between the early nineteenth century and the Second World War, when uranium production took another direction. Uranium glass today is manufactured only for specific industrial rather than decorative purposes. Tomabechi describes well the peculiar scientific properties of uranium glass, and gives a comprehensive account of the centres at which it was produced in Europe, the United States and Japan. The resplendent colour illustrations show a wide range of uranium glass products from the author's collection.

There is, however, one section of the book that I find difficult to accept. This is where it is asserted that the Romans exploited uranium glass in order to achieve a light green tint in mosaics. The evidence is said to lie in some mosaic tesserae from a villa at Cap Posillipo destroyed at the same time as Pompeii in AD 79, supposedly brought back to Oxford by the excavator R. T. Gunther in 1911.
The relevant scientific literature certainly states as much. The reality, as described to me by David Brown, my predecessor as curator of the Roman section of the Ashmolean Museum, is somewhat different.

Uranium glass first entered the picture in 1911 when it was the only material with which the Daubeny Laboratory at Oxford could replicate the effect of the Roman glass This was confirmed in the later 1960s when, at Franz Kirchheimer's suggestion, samples of fused glass preserved in test-tubes at the Ashmolean were analysed by the Max Planck Institute in Munich. None proved to be older than 1911, and none of the complete tesserae kept with them was found to contain uranium at all. The site at Cap Posillipo was also scanned with Geiger counters, and nothing of interest was registered. These researches were never published; Kirchheimer's interest seems to have waned once the Romans, and the possibility of 2,000-year-old uranium, fell out of the picture. To complicate matters further, the samples in Oxford were accidentally thrown away a few years ago, to the great disappointment of researchers who periodically come looking for them.

This is, however, simply intended to put the record straight, and in no way to belittle Tomabechi's achievement in writing the first book on a material of such scientific, social and aesthetic interest.

Michael Vickers is at the Ashmolean Museum of Art and Archaeology, University of Oxford, Oxford OX1 2PH, UK.

\section{Sensible to feeling}

\section{Stuart Sutherland}

Emotional Intelligence: Why It Can Matter More Than IQ. By Daniel Goleman. Bantam: 1995. Pp. 342. \$23.95. To be published in the United Kingdom by Bloomsbury on 10 January at $£ 16.99$.

LIKE the Bible, the literature of social psychology can provide support for any view one happens to hold: it is ridden with contradictory results and pitfalls in interpretation. For example, it is easy to assume that because parents have been beastly to a child, he or she will turn into a beastly adult, but there are two alternative explanations. First, the parents may have treated the child badly because it was fractious from an early age (and continued to be so in later life). Second, the transmission of bad behaviour may have occurred through genetic inheritance affecting both parent and child in the same way, rather than through the child's environment.

Although Daniel Goleman does not consider these alternatives, he puts forward a case for the influence of parents on personality, but he does so by selecting only studies that support his argument: there are many others that do not. For example, in a massive longitudinal study, George Vaillant found that an unhappy childhood was associated with success in later life. Goleman maintains, however, that "emotional intelligence" is an important factor in determining success. These curious buzz words mean the capacity to know and to verbalize what emotion one is feeling at a given time and why one is feeling it, the capacity to sense what emotion others are feeling and to emphathize with them, and the ability to control one's reactions when emotionally aroused. But Goleman cites no evidence that these attributes of emotional intelligence actually occur together: indeed, cold people are usually restrained.

In an ingenious experiment, Walter Mischel presented 4-year-old children with a marshmallow each, telling them that he was going on an errand and that on return he would give them another one, provided they had not already eaten the first. When they were tracked down at the age of 18, it was found that those able to resist temptation had higher examination scores than those who could not. Moreover, their success at 18 was better predicted by the marshmallow test than by conventional IQ tests, although Goleman admits that IQ is a better predictor from about the age of 8 onwards. Indeed, emotional intelligence is not the only route to success: neither Hitler nor Stalin were notable for empathy or self-restraint, but by their own lights both were successful.

Where psychology leads, can neurophysiology be far behind? Goleman mentions Antonio Damasio's exciting work showing that signals associated with emotion that arise in the prefrontal lobes are important for making decisions. He also cites evidence that visual stimuli are analysed not merely by the visual cortex but also by a fast route through the amygdala, which enables people to respond quickly to threat, either with fight or flight: the same applies to other modalities. It is the longer pathways by way of the sensory cortices that enable spontaneous outbursts of emotion to be overcome. Interestingly, lesions in the left prefrontal lobes produce anger and fear, whereas lesions in the right can give rise to extreme cheerfulness. All this may be true, but it is worth observing that despite our anatomical knowledge of the brain's pathways, nobody can explain how their working leads to the subjective emotions of fear or anger and their accompanying reactions: the sympathetic system is aroused in similar ways in both.

Perhaps the most interesting part of Emotional Intelligence is the description of the programmes devised to teach that skill, which now exist in about 20 US schools, including ones for deprived 